\title{
Functional small RNAs are generated from select miRNA hairpin loops in flies and mammals
}

\author{
Katsutomo Okamura, ${ }^{1,2,3}$ Erik Ladewig, ${ }^{1}$ Li Zhou, ${ }^{2}$ and Eric C. Lai ${ }^{1,3}$ \\ ${ }^{1}$ Department of Developmental Biology, Sloan-Kettering Institute, New York, New York 10065, USA; ${ }^{2}$ Temasek Lifesciences \\ Laboratory, National University of Singapore, Singapore 117604
}

In the canonical animal microRNA (miRNA) pathway, Drosha generates $\sim 60$ - to 70 -nucleotide pre-miRNA hairpins that are cleaved by Dicer into small RNA duplexes that load into Argonaute proteins, which retain a single mature strand in the active complex. The terminal loops of some miRNA hairpins regulate processing efficiency, but once liberated by Dicer, they are generally considered nonfunctional by-products. Here, we show that specific miRNA loops accumulate in effector Argonaute complexes in Drosophila and mediate miRNA-type repression. This was unexpected, since endogenous loading of Argonaute proteins was believed to occur exclusively via small RNA duplexes. Using in vitro assays, which recapitulate Argonaute-specific loop loading from synthetic pre-miRNAs and even single-stranded oligoribonucleotides corresponding to miRNA loops, we reveal that the loop-loading mechanism is distinct from duplex loading. We also show that miRNA loops loaded into the miRNA effector AGO1 are subject to $3^{\prime}$ resection, and structure-function analyses indicate selectivity of loop loading. Finally, we demonstrate that select miRNA loops in mammals are similarly loaded into Argonaute complexes and direct target repression. Altogether, we reveal a conserved mechanism that yields functional RNAs from miRNA loop regions, broadening the repertoire of Argonaute-dependent regulatory RNAs and providing evidence for functionality of endogenous ssRNA species.

[Keywords: miRNA hairpin loop; small RNA loading; Argonaute protein]

Supplemental material is available for this article.

Received December 19, 2012; revised version accepted February 28, 2013.

MicroRNAs (miRNAs) are a family of $\sim 21$ - to -23 nucleotide (nt) small regulatory RNAs that regulate diverse biological processes and play important roles in gene regulatory networks during development and physiology (Flynt and Lai 2008; Pelaez and Carthew 2012). Most miRNAs are transcribed by RNA polymerase II as primary hairpin-containing transcripts, followed by sequential processing by the RNase III enzymes Drosha and Dicer (Kim et al. 2009). Nuclear processing by Drosha and its partner, DGCR8, releases pre-miRNA hairpins, which are cleaved in the cytoplasm by Dicer and its partner, TRBP, into miRNA/miRNA* (star) duplexes. These duplexes are loaded into effector proteins of the Argonaute family, which select a single strand for retention in active mature regulatory complexes; the mature strand is operationally defined as the more abundant duplex species. Although this canonical miRNA processing pathway

${ }^{3}$ Corresponding authors

E-mail okamurak@tll.org.sg

E-mail laie@mskcc.org

Article published online ahead of print. Article and publication date are online at http://www.genesdev.org/cgi/doi/10.1101/gad.211698.112 generates most miRNAs, cells exploit a variety of RNA processing mechanisms to produce additional Argonautedependent small RNAs, comprising noncanonical miRNA substrates that bypass the necessity for either Drosha or Dicer (Yang and Lai 2011) or potentially even both RNase III enzymes (Maurin et al. 2012).

Argonaute proteins use structural and sequence information in the miRNA/miRNA * duplex to select an appropriate strand (Khvorova et al. 2003; Schwarz et al. 2003; Forstemann et al. 2007; Tomari et al. 2007; Czech et al. 2009; Kawamata et al. 2009; Okamura et al. 2009; Ghildiyal et al. 2010). Features of duplex loading also apply to endogenous siRNA duplexes and are recapitulated by studies of synthetic siRNA duplexes, indicating their generality. As such, small RNAs carried by Argonauteclass proteins are generally believed to derive from duplex precursors (Kawamata and Tomari 2010; Okamura 2012). It has been presumed that double-stranded precursor structures protect the RNA molecules from nonspecific degradation by abundant ssRNA-specific RNases. Consistent with this notion, ssRNAs can be loaded into purified Argonaute proteins in vitro (Martinez et al. 2002; Rivas et al. 2005), and high concentrations of ss-siRNA can over- 
come nonspecific degradation and induce sequence-specific RNAi responses in human cells or cell lysate, albeit with low efficiency (Martinez et al. 2002; Schwarz et al. 2002; Chorn et al. 2012). In addition, recent reports suggest that ssRNA loading may be a default tendency because recombinant Argonaute proteins are loaded with RNA fragments when they are expressed in Escherichia coli, whose genome lacks the miRNA machinery (Nakanishi et al. 2012), and small RNA association promotes a stable Argonaute conformation (Elkayam et al. 2012).

Nevertheless, the endogenous significance of such "bypass" loading has been questioned, since the majority of Argonaute-dependent small RNAs derive from small RNA duplexes that are loaded by the activity of duplexloading mechanisms (Kawamata and Tomari 2010). Duplex loading usually requires ATP-dependent chaperone machineries for a presumable conformational change of Argonaute proteins (Iki et al. 2010; Iwasaki et al. 2010; Johnston et al. 2010; Miyoshi et al. 2011). On the other hand, at least in in vitro systems, ssRNAs can be loaded into Argonautes in an ATP-independent manner (Liu et al. 2004; Rivas et al. 2005). However, it is noteworthy that even though piRNAs are believed to derive from singlestranded precursors, their loading requires chaperone machineries (Ishizu et al. 2012). Thus, different classes of Argonaute proteins and clients appear to have distinct requirements for the chaperone machinery.

Dicer cleavage of pre-miRNA hairpins generates three species: the mature miRNA, the miRNA ${ }^{\star}$, and an intervening terminal loop. We recently studied the regulatory roles of miRNA * strands, which were previously regarded largely as processing by-products. Although miRNA* strands accumulate to lower levels compared with their partner mature strands, they are frequently conserved and have demonstrable regulatory function in experimental assays used to define activities of mature miRNA strands (Okamura et al. 2008; Yang et al. 2011). These findings inspired us to examine miRNA loops in greater detail. Because miRNA loop regions generally do not form stable double-stranded structures, miRNA loops were assumed to be unstable after processing. Nevertheless, miRNA loop species have begun to emerge in small RNA cloning efforts as deep-sequence throughput has increased, and some miRNA loci produce a substantial number of loop reads (Ruby et al. 2007; Ghildiyal et al. 2010; Berezikov et al. 2011). This suggests that some miRNA loops are protected by RNA-binding proteins (Bajan and Hutvagner 2011; Ruwe and Schmitz-Linneweber 2012).

In this study, we demonstrate that the stability of specific miRNA loops in animal cells is due to incorporation into Argonaute complexes and that they can direct post-transcriptional repression. We support this using a combination of genome-wide analysis, functional molecular studies, and in vitro processing assays. Moreover, we show conservation of the unexpected regulatory activity of miRNA loop species from Drosophila to mammals. Overall, our results extend the range of endogenous small RNA species in Argonaute complexes and imply the existence of mechanisms that sort ssRNAs into specific miRNA/siRNA-class Argonaute complexes. The recog- nition of this pathway may be relevant for recent efforts to improve the activity of ss-siRNAs in mammalian cells (Chorn et al. 2012; Lima et al. 2012; Yu et al. 2012).

\section{Results}

Specific miRNA loop reads populate mature Drosophila Argonaute complexes

The terminal loop reads of some miRNA loci are evident in small RNA libraries, and the precise phasing of such loop reads with the termini of flanking miRNA and star species serves as especially stringent evidence for the endogenous processing of a miRNA hairpin by Dicer (Ruby et al. 2007; Berezikov et al. 2011). However, the modest recovery of terminal loop reads, with many loci lacking such reads even in very deep small RNA data sets, has suggested that they are simply by-products of miRNA biogenesis. As such, we expected such nonfunctional RNA fragments to be depleted from AGO1 immunoprecipitation libraries, relative to companion total RNA libraries.

Consistent with this scenario, likely degradation products such as fragments of rRNA or ribosomal protein mRNAs were strongly depleted in AGO1 immunoprecipitation data relative to companion total RNA (approximately fivefold to 10-fold) (Fig. 1A). Surprisingly, however, the reads mapping to mir-34 and mir-317 loop regions were enriched in AGO1 immunoprecipitation libraries |6.1-fold and 1.4-fold, respectively), similar to their mature miRNA counterparts $(0.7$-fold and 5.2-fold enrichment, respectively) (Fig. 1A). This suggested that these miRNA loops were specifically incorporated into AGO1 complexes. The read counts of these miRNA loops were not trivial, with $>42,000$ and $>20,000$ reads for mir-34 and mir-317 loops recovered, respectively, in the analyzed 252 libraries consisting of $\sim 1$ billion reads. These numbers exceed the read counts of the 105th (miR-137: 39,734 reads) and 116th (miR-978: 18,101 reads) most abundant mature miRNA species among the 245 miRBase genes, respectively (Supplemental Table S1, "Summary" spreadsheet). The highest expression of mir-34 gene was observed in fly heads, and mir-34 loop reads accumulated to 573.17 RPM /reads per million) in the wild-type head total RNA libraries. We verified the small RNA library data by showing that the endogenous mir-34 loop was readily detected by conventional Northern blotting of head total RNA and AGO1 immunoprecipitation samples (Supplemental Fig. S1).

To obtain further insight into the association of these loops with Argonaute complexes, we purified the major Drosophila somatic Argonaute complexes (AGO1 and AGO2) from S2- $\mathrm{R}^{+}$cells expressing Flag-tagged AGO2 protein using anti-AGO1 and anti-Flag antibodies (Czech et al. 2008). In general, mature miRNAs are enriched in AGO1 complex, while endo-siRNAs are sorted to AGO2 complexes (Miyoshi et al. 2005; Czech et al. 2008; Kawamura et al. 2008; Ghildiyal et al. 2010). We confirmed efficient purification of these complexes using miRNA (bantam) and endo-siRNA (hp-CG4068B) probes, which detected AGO1- and AGO2-enriched species, respectively (Fig. 1B). Under these experimental conditions, we ob- 
A

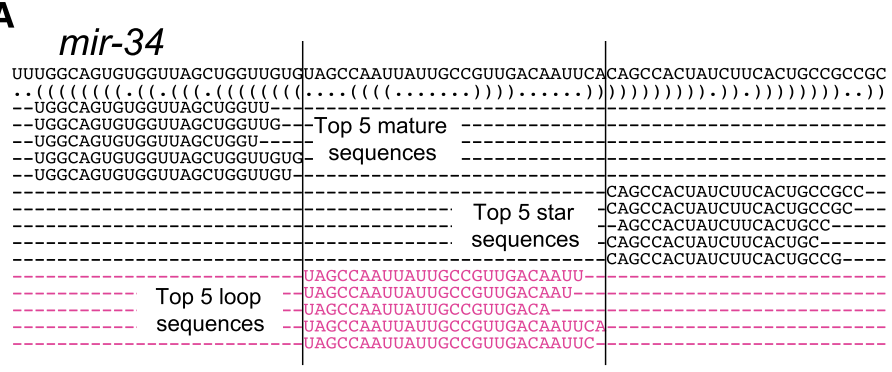

mir-317

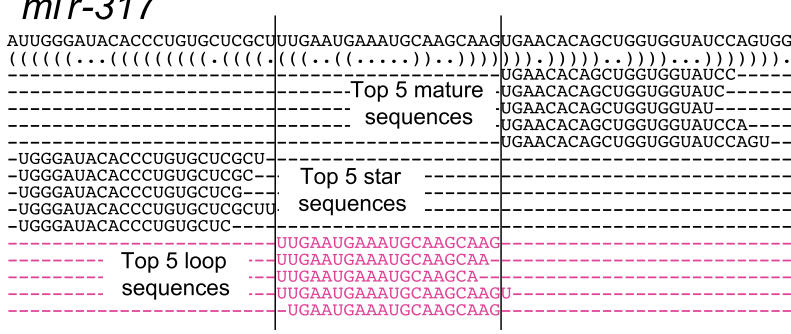

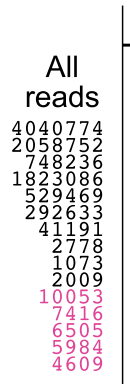

reads per million

Ovary Ovary

total AGO1

5991:9 3279.0

$2805: 6 \quad 3606: 5$

$\begin{array}{rl}2883: 9 & 3626: 9 \\ 21 & 26.1 \\ 21 & 275:\end{array}$

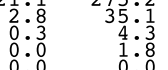

0

$\begin{array}{rr}2: 4 & 17: 0 \\ 1 & 7: 1 \\ 0 & 7: 9 \\ 0: 9 & 9: 9\end{array}$

reads per million

All

Ovary Ovary

total AGO1

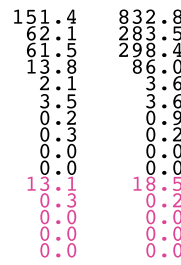

$\begin{array}{lll}\text { RpL mRNAs } & 811.9 & 81.9\end{array}$

rRNAs $4280.7 \quad 855.0$

B
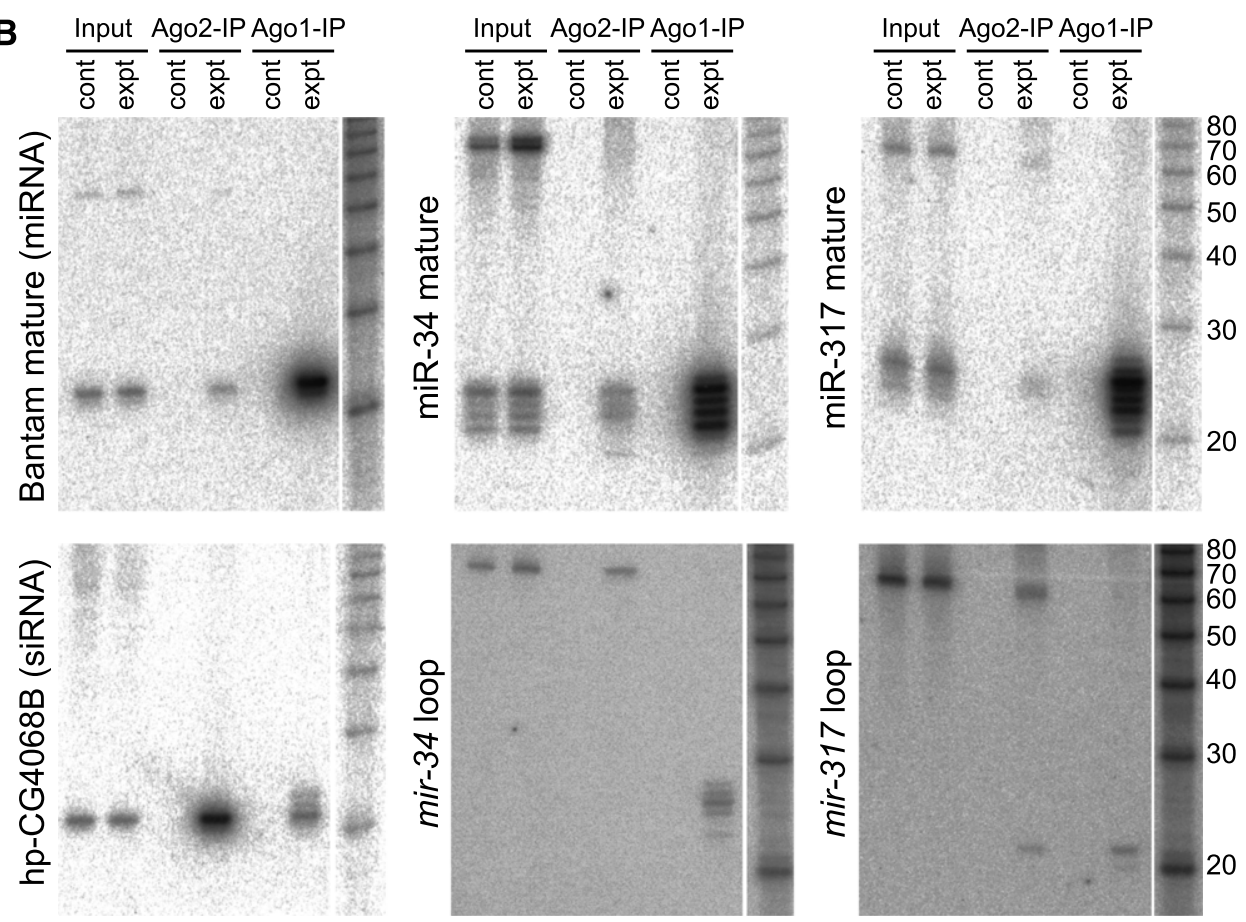

Figure 1. miRNA loops in Argonaute complexes. (A) Small RNA reads mapping to the miRNA hairpin regions of mir-34 and mir-317. The five most abundant loop, mature, and star reads are shown. In the "total" column, the numbers report raw read numbers in all of the fly small RNA libraries analyzed in this study (listed in Supplemental Table S3). The numbers in the "ovary AGO1-IP" and "ovary total" columns show read counts normalized by total number of mapped reads per miRNA (reads per million) in the libraries prepared from purified AGO1 complex or total RNA samples from ovaries. Note that loop small RNA species and mature miRNAs exhibit similar enrichment in the AGO1 complexes. The 5' ends of miRNA loops are more precisely defined than their 3 ' ends, resembling the Nibbler-mediated resection after miRNA loading. $(B)$ Detection of miRNA loop species loaded to the Argonautes by Northern blotting. Flag-tagged AGO2 and endogenous AGO1 complexes were purified from S2- $\mathrm{R}^{+}$cells stably transfected with the Flag-AGO2 plasmid. Coprecipitated RNA was analyzed by small RNA Northern blotting. The probes detected pre-miRNAs and processed loop or mature species. mir-317 and mir-34 loops were enriched in the AGO1 complex, while they were undetectable in the input samples. mir-317 loop was also detectable in the AGO2 complex. A canonical miRNA (bantam) and an endo-siRNA (hp-CG4068B) were enriched in the AGO1 and AGO2 complexes, respectively, indicating successful purification of these complexes. 
served endogenous mir-34 and mir-317 loops in Argonaute complexes. Curiously, these miRNA loops were differentially sorted into AGO1 and AGO2 complexes, with the mir-34 loop detected more strongly in AGO1 than the mir-317 loop. In contrast, we observed significant amounts of the mir-317 loop in the AGO2 complex, whereas the mir-34 loop was barely detected in this complex. Differential loop sorting was not correlated with sorting of the mature strands of these miRNAs, both of which were enriched in AGO1, as expected for most mature miRNA species (Fig. 1B). The distinct sorting behavior of the mir-34 and mir-317 loops and the distinct sorting behavior of the mature and loop species of mir-317 rule out the trivial possibility that miRNA loops in Argonaute immunoprecipitates merely correspond to intermediate processing complexes.

Further analysis supported loading of miRNA loops into mature Argonaute complexes. It was recently shown that miRNA 3' ends are susceptible to trimming by the Nibbler $3^{\prime} \rightarrow$ 5' exonuclease (Han et al. 2011; Liu et al. 2011; Westholm et al. 2012), providing a molecular basis for the higher precision of $5^{\prime}$ miRNA ends relative to their $3^{\prime}$ ends (Ruby et al. 2007; Wu et al. 2007; Seitz et al. 2008). Small RNA sequence data revealed that the $5^{\prime}$ ends of mir-34 and mir-317 loops were defined much more precisely than their 3' ends (Fig. 1A). Northern analysis confirmed that both mir-34 and mir-317 loops were trimmed in AGO1 complexes, and this was particularly obvious for the mir34 loop, whose predicted size after Dicer cleavage was 27 nt. This was consistent with the observation that longer single-stranded species, including mature miR-34 and miR-317 themselves, are preferentially subject to 3 ' resection in AGO1 (Han et al. 2011; Liu et al. 2011; Westholm et al. 2012). In contrast, the mir-317 loop loaded in AGO2 was resistant to such trimming. Nibbler is specific to single-stranded species loaded in AGO1 and is blocked by the 2' methyl group deposited at the $3^{\prime}$ nucleotide of AGO2-loaded species by Hen1 (Horwich et al. 2007; Han et al. 2011). Consistent with the distinct 3 ' structures of AGO1- and AGO2-loaded species, we observed that only mir-317 loop species coprecipitated with the AGO2 complex are resistant to oxidation by $\mathrm{NaIO}_{4}$ followed by $\beta$ elimination, which selectively oxidizes vicinal $2^{\prime}$ and $3^{\prime}$ hydroxyl groups at the $3^{\prime}$ nucleotide and removes an oxidized 3' nucleotide (Fig. 2A). We conclude that endogenous mir-34 and mir-317 terminal loops populate mature Argonaute complexes in Drosophila.

\section{Regulatory activity of mir-34 and mir-317 loops}

Since these miRNA loops accumulate to lower levels than their companion mature species, their detection in Argonaute complexes did not necessarily imply their relevance for target regulation. To assess their capacity to direct functional repression, we turned to the well-established luciferase sensor assay. We constructed luciferase reporters bearing sequences perfectly complementary to the mature and loop sequences of mir-34 and mir-317. As expected, overexpression of cognate miRNAs strongly repressed expression of mature miR-34 or miR-317 target sensors, while noncognate miRNA overexpression had little or no effect (Fig. 2B). Similarly, when we overexpressed pri-mir34 , we detected specific repression of the mir-34 loop sensor by $\sim 50 \%$ (Fig. $2 B$ ). Overexpression of pri-mir-317 caused even stronger repression of its cognate loop sensor (Fig. 2B). Animal miRNAs are usually partially complementary to their target mRNAs and trigger translational repression or mRNA destabilization. We mimicked the partially complementary targets by introducing mismatches in the target sequences complementary to the central regions (10th-12th nucleotides counting from the $5^{\prime}$ end) of miRNA loop sequences. The luciferase sensors bearing partially complementary sequences could be repressed by expression of cognate primary miRNA constructs. We conclude that multiple miRNA loop species can regulate both perfectly complementary and bulged targets, similar to mature miRNAs.

Altogether, these observations reveal a novel mechanism of small RNA loading that sorts terminal loops of miRNA hairpins into specific Argonaute complexes. The
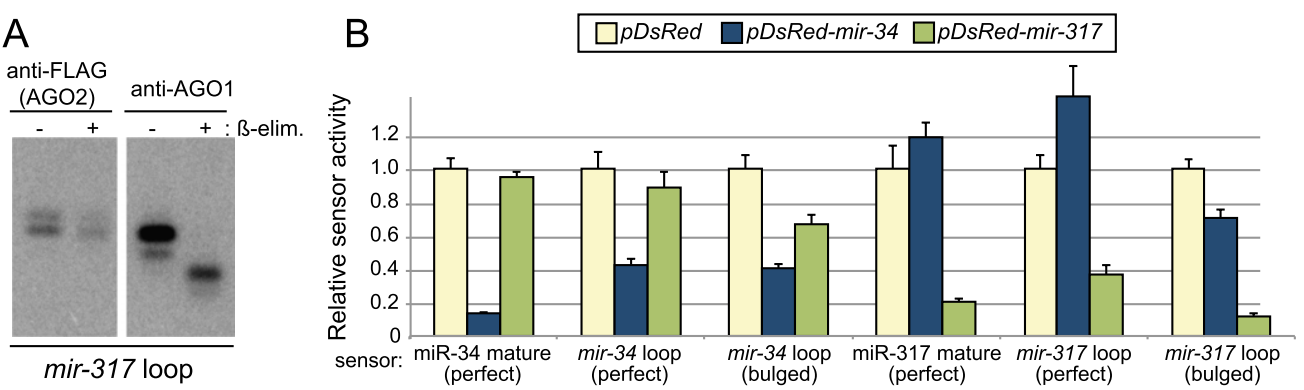

Figure 2. Proper loading of miRNA loops to the Argonautes. (A) 3' modification of AGO2-loaded mir-317 loop. mir-317 was overexpressed in $\mathrm{S} 2-\mathrm{R}^{+}$cells expressing Flag-AGO2, and AGO1 and Flag-AGO2 complexes were isolated by immunoprecipitation. Coprecipitated RNA was subjected to $\beta$ elimination, and mir-317 loop was detected by Northern blotting. The AGO2-loaded mir-317 loop species was resistant to $\beta$ elimination, whereas the AGO-1-loaded species was fully sensitive. $(B)$ Regulatory activity of the miRNA loop species. Sensor plasmids containing two perfectly complementary targets to the loop or mature sequences of mir-34 and mir-317 cloned downstream from Renilla luciferase were used. Sensor plasmids were cotransfected with ub-Gal4 and UAS-DsRed or UAS-DsRed-miRNA plasmids as indicated. The values are shown as the mean of the relative ratios of Renilla/firefly luciferase sensor values normalized by the values of DsRed alone, and error bars represent the standard deviations. 
association of miRNA loops with Argonaute did not represent fortuitous binding, since we observed differential sorting and subsequent $3^{\prime}$ trimming or $3^{\prime}$ modification of miRNA loops in AGO1 and AGO2 complexes. Furthermore, miRNA loops were capable of guiding specific target repression. Overall, these data were surprising because small RNA sorting in Drosophila was believed to depend on their precursor duplex structures and 5' nucleotides (Schwarz et al. 2003; Tomari et al. 2007; Czech et al. 2009; Kawamata et al. 2009; Okamura et al. 2009; Ghildiyal et al. 2010). Neither of the known sorting rules explains their sorting patterns, as both mir-34 and mir-317 loops derive from single-stranded portions of premiRNA hairpins and have $5^{\prime}$ uridine. This suggests that small RNA biogenesis pathways are more complex than currently appreciated.

\section{Loading of miRNA loop species from a synthetic pre-miRNA in vitro}

The $5^{\prime}$ ends of most mir-34 and mir-317 loop reads coincide with the predicted Dicer-1 cleavage site (Fig. 1A), suggesting that they are Dicer products derived from pre-miRNAs. However, we could not formally exclude the possibility that Argonaute-loaded mature and loop species might be products of different precursor molecules. For example, the orthologs of some nematode miRNA loci unexpectedly adopt different folds involving distinct pre-miRNAs, which pair a given mature miRNA arm to either upstream or downstream transcribed regions (de Wit et al. 2009). To confirm that the Argonaute-loaded loops were indeed produced from the same defined pre-miRNA species, we developed an in vitro system that recapitulates loop loading (Fig. 3A).

We prepared synthetic pre-mir-34 hairpins labeled with ${ }^{32} \mathrm{P}$ at the $5^{\prime}$ end or within the terminal loop. We then incubated these with lysate from $\mathrm{S}^{2} \mathrm{R}^{+}$cells expressing Flag-HA-AGO2, which allowed us to purify either Argonaute complex using AGO1 or Flag immunoprecipitation. Upon incubation of the 5'-labeled pre-miRNA with $\mathrm{S} \mathrm{R}^{+}$lysate, we observed dicing into the expected $\sim 24$-nt mature species, which associated with AGO1. This confirmed that our in vitro system reliably recapitulated miRNA processing and loading processes. Under the same experimental condition, loop-labeled pre-mir-34 was efficiently processed, judging by the reduction of pre-miRNA substrate and concomitant appearance of the 27-nt loop (Fig. 3B, input lanes). Although the efficiency of loop loading into AGO1 was lower than the mature miR-34, we nonetheless readily detected the mir-34 loop in the immunopurified AGO1 complex. With longer reaction time points, the 27-nt mir-34 loop was further processed into shorter species (Fig. 3B), resembling the reported post-loading trimming of select miRNA species by Nbr (Han et al. 2011; Liu et al. 2011; Westholm et al. 2012).

To further confirm that miRNA loops are products of miRNA processing enzymes, we knocked down the RNase III enzymes Drosha and Dcr-1 and their partners, Pasha and loqs, in S2- $\mathrm{R}^{+}$cells stably transfected with an inducible mir-34 overexpression plasmid. mir-34 loop production was reduced after knocking down the miRNA factors, and the degree of its reduction correlated with mature miR-34 production (Fig. $3 \mathrm{C}$ ). Therefore, miRNA loop species are processed by the canonical miRNA processing pathway.

We next asked whether the mir-34 loop processed in the lysate was specifically sorted to the AGO1 complex, as observed with its endogenous counterpart. After in vitro processing of pre-mir-34, we purified AGO1 and AGO2 complexes. Endogenous small RNAs remained associated with the specific Argonaute proteins, suggesting that the exchange of small RNAs between two distinct Argonaute complexes was limited, if present at all, under this in vitro condition (Fig. 3D). As predicted, mature miR-34 processed in vitro was enriched in the AGO1 complex (Fig. 3D, top left panel). The mir-34 loop processed in vitro was also more efficiently loaded to the AGO1 complex, whereas the mir-317 loop was enriched in the AGO2 complex, as with their respective endogenous loop sorting patterns (Fig. 3D [top right panel], E). These data confirm that miRNA loop species can be generated from defined pre-miRNAs and loaded to specific Argonaute complexes in vitro. Surprisingly, we observed similar sorting patterns with preprocessed synthetic oligoribonucleotides corresponding to mir-34 and mir-317 loop sequences (Fig. 3F). These results suggested that Drosophila AGO1 and AGO2 possess inherent preferences for particular ssRNA molecules. The similar sorting ratios between synthetic miRNA loops and miRNA loops processed from pre-miRNA hairpins further suggest that miRNA loop species were loaded as ssRNA molecules and not as duplex structures.

The in vitro loading system offered an opportunity to gain deeper insight into the miRNA loop loading mechanism. We asked whether miRNA loop loading depends on the HSP (heat-shock protein) chaperone machineries. It has been proposed that duplex loading requires ATP hydrolysis by the chaperone machineries in order for Argonautes to undergo a conformational change to accommodate duplex RNA molecules. In contrast, single-stranded small RNAs can be incorporated independently of HSP activity in vitro. We hypothesized that the miRNA loops might be loaded to Argonaute proteins in a chaperone-independent manner. To inhibit Hsc70 activity, we added PES (2-phenylethynesulfonamide) to the lysate. In the presence of PES, loading of miRNA duplex to Argonaute complexes was strongly inhibited (Fig. 3G, top panel). Under the same condition, we tested whether miRNA loops were loaded to the Argonaute complexes. While we observed detectable reduction of miRNA loop loading, we could still observe loop species loaded in the Argonaute complexes. Loading of miRNA loops to AGO2 was especially less sensitive to PES. These results suggested that miRNA loops are loaded to Argonaute proteins in a mechanism that is distinct from the duplex-loading machinery. The reduction of miRNA loop species in Argonaute complexes in the presence of PES might be consistent with previously observed slight reduction of ssRNA-loading activity in the presence of PES (Iwasaki et al. 2010). In summary, all of the results suggested that miRNA loops are loaded as ssRNA molecules by noncanonical small RNA-loading mechanisms. 

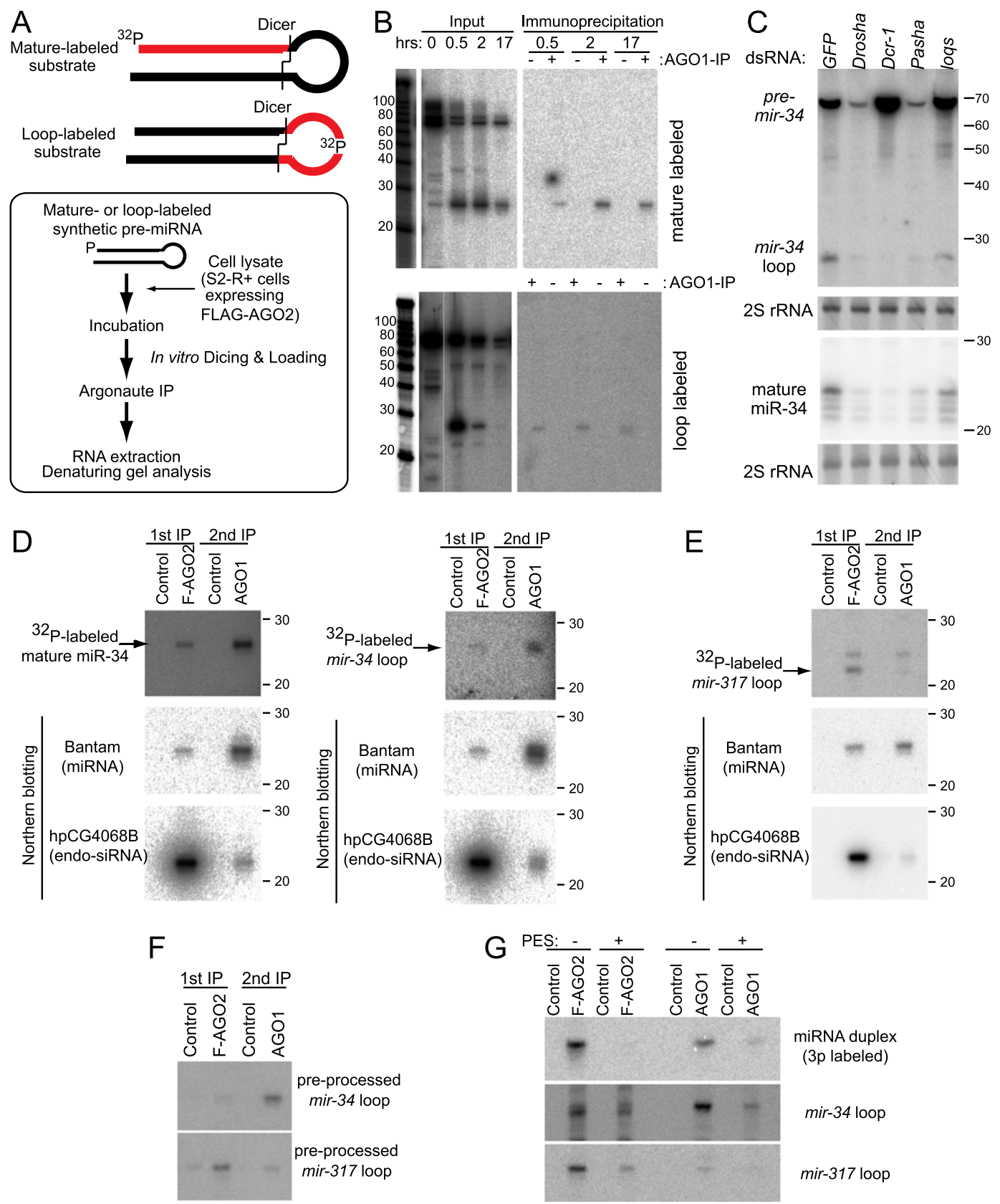

Figure 3. Mechanism of miRNA loop loading. $(A)$ A schematic representation of the experimental design. Synthetic pre-miRNAs were labeled at the $5^{\prime}$ ends or in the loop regions to monitor the processed $5 \mathrm{p}$ mature or loop species. The labeled pre-miRNAs were incubated in cell lysate prepared from S2- $\mathrm{R}^{+}$cells expressing Flag-AGO2, and the Argonaute complexes were then purified by anti-Flag and antiAGO1 antibodies. Coprecipitated radioactive RNA molecules were analyzed by autoradiography. (B) Coprecipitation of in vitro processed mature and loop species with AGO1. Radiolabeled pre-miRNAs were incubated in cell lysate for 0.5, 2, or $17 \mathrm{~h}$, and the AGO1 complex was purified from the lysate. Each set of mature or loop panels was cropped from the same exposure of the same gel. Note that the mir-34 loop was converted to heterogeneous species after a 17-h incubation resembling the endogenous mir-34 loop detected by Northern blotting. $(C)$ mir-34 loop production is dependent on the canonical miRNA pathway. The miRNA processing factors were depleted by RNAi from S2- $\mathrm{R}^{+}$cells overexpressing mir-34. Small RNA production was monitored by Northern blotting using probes against the mir34 loop or mature miR-34 species. 2S rRNA was detected by ethidium bromide for loading control. $(D)$ The in vitro processed mir-34 loop was enriched in AGO1. The mir-34 loop product processed in vitro was more abundant in the AGO1 immunoprecipitate than in the FlagAGO2 complex, whereas Northern blotting analysis of endogenous small RNAs indicated successful purification of both complexes. (E) The in vitro processed mir-317 loop was enriched in AGO1. In contrast to the mir-34 loop species, the mir-317 loop species was sorted to the AGO2 complex, resembling the sorting of the mir-317 loop in vivo. (F) Single-stranded preprocessed loop RNA species can be sorted to two Argonautes. The in vitro loading assay was performed with 5' labeled synthetic RNA oligonucleotides with the mir-34 or mir-317 loop sequence. Synthetic mir-34 and mir-317 loops were sorted to the Argonaute complexes with ratios similar to those observed with the loops processed from pre-miRNAs in vivo (Fig. 1B) or in vitro $(D, E)$. $(G)$ Effect of an hsp70 inhibitor PES on miRNA loop loading. The in vitro loading assay was performed using a miRNA duplex (mir-276a duplex labeled with the $5^{\prime}$ end of the 3p strand) or loop-labeled premir-34 or pre-mir-317. The mir-276a duplex was analyzed on a native gel, and miRNA loop species were analyzed on a urea denaturing gel. miRNA duplex loading is inhibited by PES, as shown with the mir-276a duplex; however, loading of miRNA loops was detectable in both the AGO1 and AGO2 complexes, although the loading efficiency was reduced by PES. The reduction of miRNA loop loading might be consistent with the previously reported slight reduction of ssRNA loading to AGO1 (Iwasaki et al. 2010). 


\section{Structural and sequence requirements for miRNA loop loading}

Most procedures for small RNA cloning select for $>18-n t$ small RNAs on account of the fact that substantially shorter reads usually do not map uniquely. Consequently, small RNAs shorter than this range are not expected to be included in most available libraries. While the lengths of the abundant miRNA loop species happen to fall in the range of miRNA cloning, the majority of Drosophila premiRNAs have shorter loops (median $=17 \mathrm{nt}$, calculated as the regions inferred to be released upon Dicer-1 cleavage, based on $\sim 2$-nt $3^{\prime}$ overhangs formed between the $5^{\prime}$ ends of major miRNA-3p species and the $3^{\prime}$ ends of miRNA-5p species) (Supplemental Table S1, "Summary" spreadsheet). For example, the bantam loop is only $14 \mathrm{nt}$ in length. Thus, the representation of loop reads in small RNA libraries has likely been biased by size selection during cloning procedures.

We asked whether the mir-34 loop could be loaded to AGO1 if it was shorter. We prepared variant pri-mir-34 constructs with serial 3 ' truncation of the loop region from the initial $27 \mathrm{nt}$ to $22 \mathrm{nt}$ and $15 \mathrm{nt}$ (Fig. 4A). We first verified that these variants were still competent to generate active mature miR-34 by cotransfecting them with the miR-34 luciferase sensor. Indeed, there was no appreciable difference in their repression activities (Fig. 4A). The 22-nt variant mir-34 loop was still active similarly to the wildtype loop; however, the 15-nt loop variant did not direct efficient target repression (Fig. 4A). We complemented the sensor test using the in vitro loop-loading assay. We synthesized a mutant pre-mir-34 bearing a 15-nt loop and labeled this at the $5^{\prime}$ end or within the loop region. As expected, the mature miR-34 species from the mutant premir-34 was efficiently produced and loaded to AGO1, confirming proper folding and dicing of this pre-miRNA (Fig. 4B, left panels). When the mutant pre-miRNA was labeled in the loop region, this hairpin was also efficiently processed but did not produce loop species loaded to the AGO1 complex (Fig. 4B, right panels). This suggests that sufficient length of the miRNA loop is an important determinant of the loop-loading efficiency.

We performed additional tests to see whether any loop species of appropriate length could be loaded to the
A
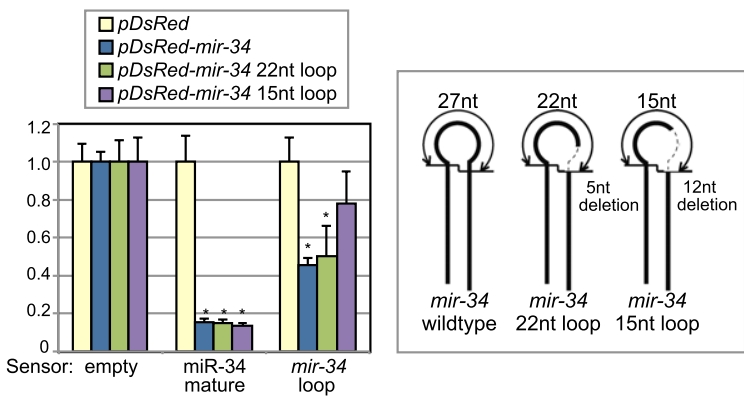

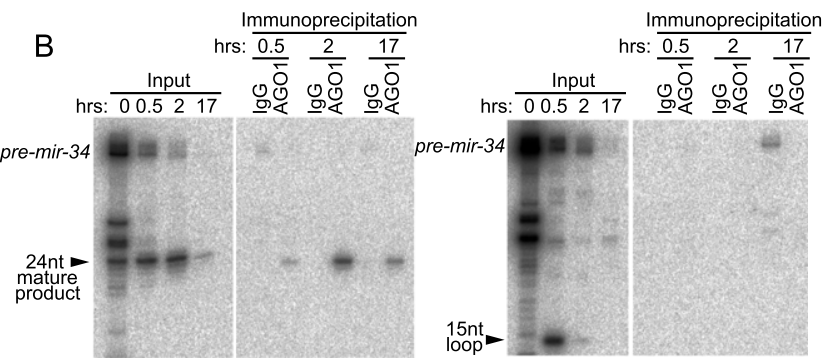

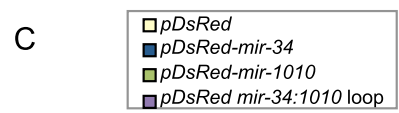

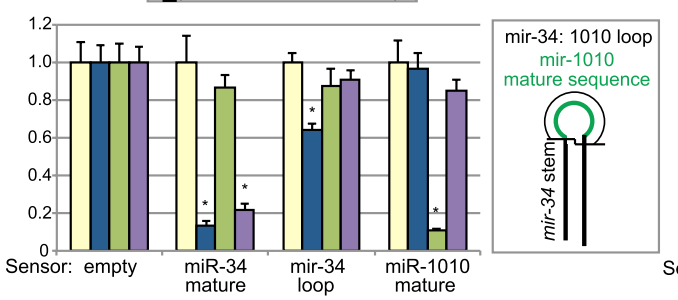

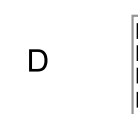
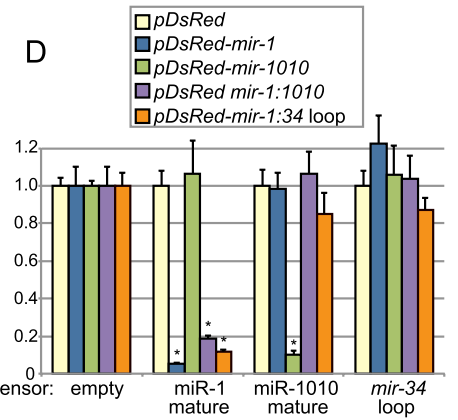

$\mathrm{E} \mathrm{apDsRed}^{p D s e d}$ 口pDsRed-mir-34 (D. mel) 口pDsRed-mir-34 (cognate)
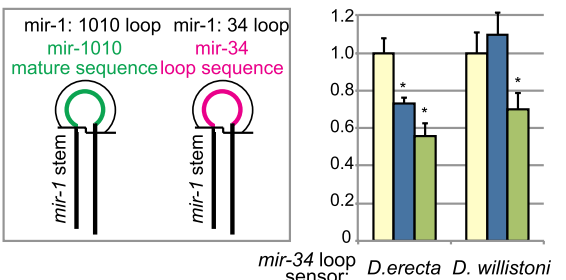

Figure 4. Specificity of miRNA loop loading. (A) pri-mir-34 variants bearing shorter (22-nt and 15-nt) loop regions were overexpressed in S2- $\mathrm{R}^{+}$cells along with the luciferase reporter bearing a perfectly complementary mir-34 loop target sequence. Although the mature sensor was repressed similarly by overexpression of these three pri-mir-34 variants, the loop target was not efficiently repressed by the mir-34 15-nt loop mutant. Asterisks indicate statistical significance compared with the values of the same sensor with pDsRed empty vector $(P<0.001$, Student's $t$-test). (B) Inefficient incorporation of a 15-nt loop into AGO1 in vitro. The mir-34 mutant (15-nt loop) was labeled in its loop regions and used for the in vitro loading assay. While the mutant mir-34 (15-nt loop) was efficiently processed, the loop product was not coprecipitated with AGO1, suggesting that shorter loops are not suitable for stable loading to AGO1. Each set of mature or loop panels was cropped from the same exposure of the same gel. $(C, D)$ Luciferase assays were done with reprogrammed constructs based on the mir-34 hairpin $(C)$ or mir-1 hairpin $(D)$. The loop regions of these hairpins were replaced with the mature miR-1010 (1010-loop constructs) or mir-34 (34-loop construct) loop. Asterisks indicate statistical significance compared with the values of the same sensor with pDsRed empty vector $(P<0.001$, Student's $t$-test $)$. (E) Conservation of the mir-34 loop loading in multiple Drosophilids. Perfectly complementary targets for the loops of mir-34 homologs in two Drosophila species (Drosophila erecta and Drosophila willistoni) were cloned in the $3^{\prime}$ untranslated region (UTR) of the luciferase gene in the psiCHECK vector. Target expression was repressed when the mir-34 gene cloned from the cognate species was overexpressed. Asterisks indicate statistical significance compared with the values of the same sensor with pDsRed empty vector $(P<0.001$, Student's $t$-test $)$. 
Argonaute. We replaced the mir-34 loop sequence with the sequence of mature miR-1010, a miRNA not detectably expressed by S2 cells. Although this reprogrammed construct efficiently repressed the mature miR-34 sensor, it failed to repress a miR-1010 sensor (Fig. 4C). Furthermore, when the loop region of pre-mir-1 was replaced with the miR-34 loop sequence, overexpression of this chimeric miRNA could only repress the miR-1 target but not the mir-34 loop target (Fig. 4D). These tests suggested that neither loop length nor sequence is sufficient to promote efficient Argonaute loading.

Because we observed a strict selectivity of miRNA loop loading for a subset of miRNA species, we were interested to determine whether the loops of mir-34 homologs from other species have regulatory activity. Although the mature and star sequences of mir-34 are identical across sequenced Drosophila species, the loop region has diverged significantly (Supplemental Fig. S2). We cloned mir-34 homologs from two Drosophila species with distinct loop sequences (Drosophila erecta and Drosophila willistoni) and constructed luciferase sensors bearing perfectly loopcomplementary targets. We cotransfected S2- $\mathrm{R}^{+}$cells with these sensors and either cognate or Drosophila melanogaster pri-mir-34 plasmids. In both cases, the loop sensors were repressed by mir-34 from the cognate species (Fig. 4E). Overexpression of D. melanogaster mir-34 moderately repressed the $D$. erecta loop sensor but did not substantially repress the more diverged $D$. willistoni mir-34 loop sensor (Fig. 4E).

These results provide evidence that loop loading of mir-34 has been conserved in different Drosophila species despite divergence of the loop sequence itself. This may be correlated to the slightly higher conservation of the $5^{\prime}$ end of the mir-34 loop (Supplemental Fig. S2A) along with the potential contribution of the remainder of the conserved miR-34/star sequences. However, our other structural manipulations show that neither the pre-miRNA stem nor loop sequence nor length are sufficient to determine effective loading of miRNA loops, suggesting that a combination of these features is necessary.

\section{Genome-wide analysis reveals differential loop selectivity of Drosophila AGO1 and AGO2}

Given that miRNA loop loading is a selective process, we were interested in understanding this at the genomewide level. To this end, we analyzed our previous total S2 Drosophila small RNA data (Okamura et al. 2009) and compared them with new small RNA libraries that we prepared from AGO1 and AGO2 complexes purified from S2- $\mathrm{R}^{+}$cells expressing Flag-tagged AGO2 protein. These contain 19,981,384 and 12,405,649 reads that map to the Drosophila genome, respectively. We tabulated the numbers of small RNA reads mapping to mature, star, and loop regions of all miRBase miRNAs (Supplemental Table S1). We observed that a limited set of miRNA genes contributed the majority of loop read counts in S2 cells (Fig. 5A, left panel). Notably, analysis of AGO1 immunoprecipitation reads showed that only mir-34 and mir-317 loops were enriched in this complex, while other miRNA loops de- tected in total RNA were depleted from AGO1 (Fig. 5A, middle panel). This suggested that other miRNA loops either exist in a free state, perhaps awaiting their degradation, or are incorporated in other complexes. In fact, many of these miRNA loops were reciprocally enriched in the AGO2 complex, and this data set included a number of other miRNA loops (Fig. 5A, right panel).

We confirmed these trends by analyzing published head small RNA libraries (Ghildiyal et al. 2010) that enrich for AGO1- or AGO2-loaded species (Fig. 5B). We conclude that AGO1 strictly selects a few miRNA loops, whereas the preference by AGO2 is less stringent. The preference could not be explained by known small RNA preferences by the Argonautes, while the $5^{\prime} \mathrm{U}$ enrichment is consistent with the previous observation with miRNA or siRNA strands (Ghildiyal et al. 2010). For comparison, we note that the mir-285 hairpin is predicted to produce a $28-\mathrm{nt}$ loop with a $5^{\prime} \mathrm{U}$, but its loop appears to be excluded from the AGO1 complex (Fig. 5B), in stark contrast to the mir-34 loop that has a $5^{\prime} \mathrm{U}$ with a similar length.

Because some miRNA loop species were present in the AGO2 complex, we were interested in its loading mechanism. It has been well established that the Dcr-2/R2D2 heterodimer complex is essential for loading of exogenous and endogenous siRNAs and miRNA* strands to the AGO2 complex (Liu et al. 2003; Tomari et al. 2004; Marques et al. 2010; Okamura et al. 2011). We tested whether these miRNA loop species are also reduced in the AGO2 complex in the r2d2 mutant. Curiously, our library analysis clearly showed that the loop species are overrepresented in the oxidized libraries prepared from the r2d2 mutant (Fig. 5B). We interpret that their overrepresentation is caused by the reduction of miRNA and miRNA * reads that require the Dcr-2/R2D2-loading complex for loading to AGO2. Because we normalized the read number against the total miRNA reads in this analysis (see the Materials and Methods), one should expect overrepresentation of small RNAs loaded to AGO2 independently of the loading complex in the oxidized library from the $r 2 \mathrm{~d} 2$ mutant. We conclude that, unlike small RNA duplexes, miRNA loops can be loaded to AGO2 in the absence of the Dcr-2/R2D2-loading factor.

In summary, genome-wide analysis revealed that a subset of miRNA loop species is selected for loading and that AGO1 and AGO2 seem to have distinct loop preferences. Strikingly, loading of the loops into AGO2 appears independent of the normal loading machinery, suggesting that this process occurs by a noncanonical loading mechanism.

\section{Conservation of miRNA loop loading in mammals}

We were motivated to assess whether the loading of miRNA loops was conserved between insects and mammals. We analyzed published small RNA libraries prepared from Argonaute complexes purified from mouse brains and skin (Fig. 5C,D; Supplemental Table S3; He et al. 2012; Wang et al. 2012). As in Drosophila, we observed that a subset of miRNA loops are indeed incorporated into Argonaute complexes from mouse brains and skin. How- 
Okamura et al.
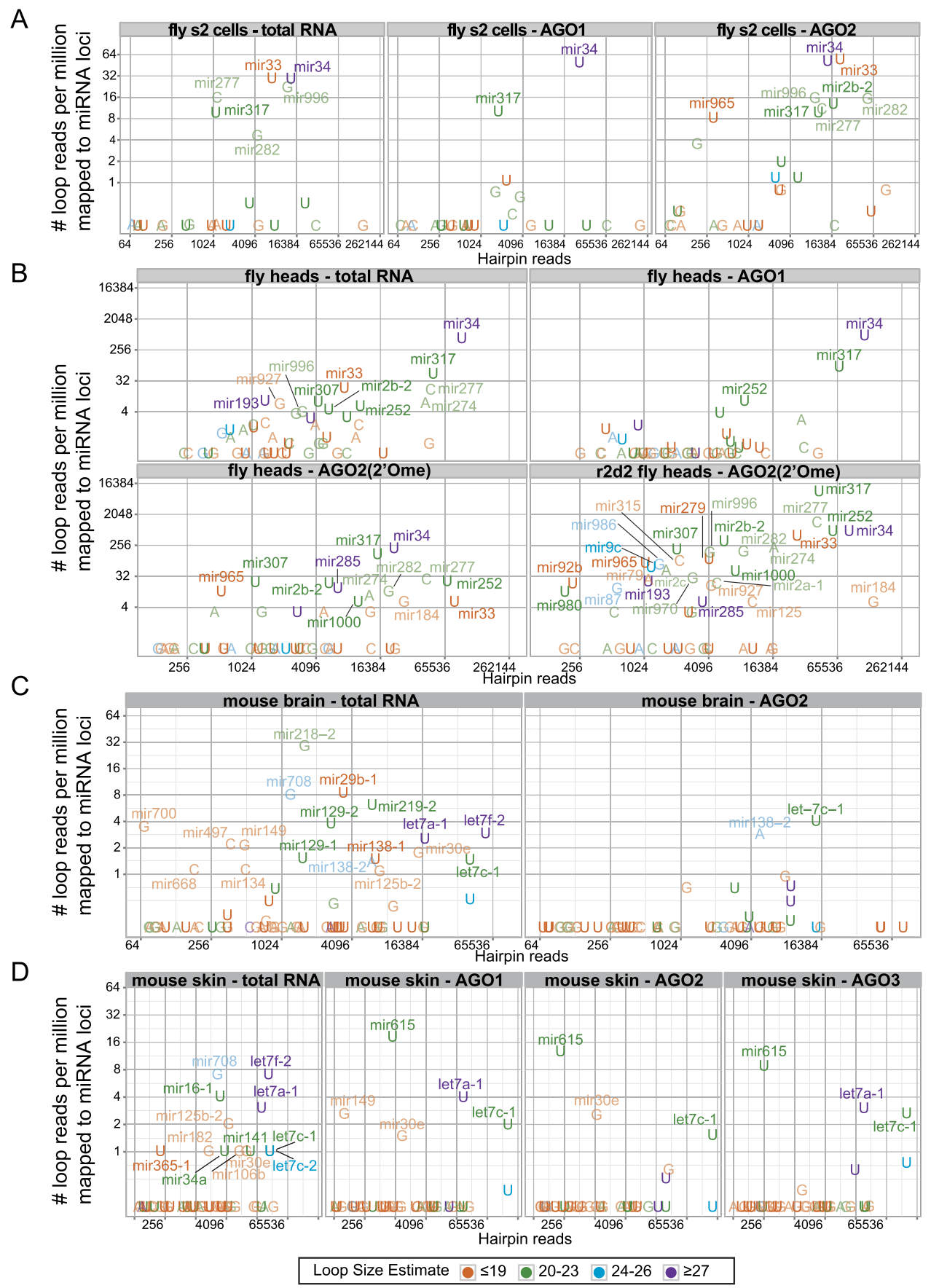

Figure 5. Genome-wide analysis of miRNA loop loading in D. melanogaster and Mus musculus. $(A-D)$ miRNA loop read counts (Y-axis) and counts of reads from the miRNA hairpin ( $X$-axis) were plotted, and A, $\mathrm{U}, \mathrm{G}$, and $\mathrm{C}$ show the predicted $5^{\prime}$ nucleotides of the miRNA loop species. The points are colored according to the estimated size of the miRNA loop. Small RNA libraries prepared from S2 cells $(A)$, fly heads $(B)$, mouse brains $(C)$, and mouse skin $(D)$ were analyzed. Read counts were normalized by number of total miRNA reads, and miRNAs with $<0.25$ RPM hairpin reads are shown at the bottom of the charts. miRNA genes with loops beginning with $5^{\prime} \mathrm{U}$ are labeled with brighter colors. Those loci whose reads per million are less than four $(A, B)$ or less than one $(C, D)$ were not labeled to keep the plot more clear.

ever, in contrast to the fly system, we did not observe an overall difference of miRNA loop species between the three Argonaute complexes analyzed in these previous studies. This is consistent with the notion that different mammalian Argonaute proteins incorporate a similar set of small RNAs (Liu et al. 2004; Meister et al. 2004; AzumaMukai et al. 2008; Ender et al. 2008).

To determine whether miRNA loop reads found in total RNA library samples also showed the signature of Argonaute loading, we analyzed nucleotide heterogeneity 
at the $5^{\prime}$ and $3^{\prime}$ termini of miRNA loop reads. It is known that the $3^{\prime}$ ends of miRNAs loaded into the Argonaute complexes are frequently modified by untemplated nucleotide addition and nucleotide trimming. As a result, $3^{\prime}$ ends of miRNAs are often more heterogeneous than the 5' ends (Ameres et al. 2010; Burroughs et al. 2010; Han et al. 2011; Liu et al. 2011; Westholm et al. 2012). We confirmed that both the mature and star species were more heterogeneous at their $3^{\prime}$ ends than at their $5^{\prime}$ ends (Fig. 6A,B; Supplemental Tables S1, S2). Similarly, we observed that miRNA loop species exhibit higher heterogeneity at their $3^{\prime}$ ends than at their $5^{\prime}$ ends in both fly and mouse libraries. This provides additional evidence that loop species reside in mature Argonaute complexes.

We chose a few miRNAs that give rise to significant numbers of miRNA loop reads for further experimental analysis. We overexpressed the selected miRNA genes in HeLa cells together with myc-tagged human Ago2 plasmid (Liu et al. 2005) and purified myc-Ago2 complexes using anti-myc antibody. All of the mature miRNA species were detected in the Ago2 complex, as expected. We observed very efficient (mir-34a, mir-615, let-7a-2, and let-7c) or modest (mir-30e) coprecipitation of miRNA loops with the Ago2 complex (Fig. 7A,B). We failed to detect loading of mir-1226 loop species even though the loop production is detected in the input samples (Fig. 7B). In contrast, mature miR-1226 species were not detected in the input sample, even though they were efficiently enriched in the immunoprecipitation sample, indicating the high specificity and efficacy of our immunoprecipitation procedure. These results suggested the existence of a selective loop-loading mechanism in mammals.

Because human Ago2 is known to associate with the Dicing complex (Chendrimada et al. 2005; Maniataki and Mourelatos 2005), it was conceivable that miRNA loops detected in Ago2 complexes represented intermediate products present in the Dicer-Ago2 complex. For example, under mild detergent conditions (Hepes-NP40 buffer), Dicer is coprecipitated with myc-Ago2 (Fig. 7C). However, we isolated Ago2 immunoprecipitates for Northern analysis using more stringent conditions (RIPA buffer), which clearly dissociates Dicer from myc-Ago2 (Fig. 7C). Therefore, the association of miRNA loops with Ago2 was not due to coprecipitated Dicer.
To verify that miRNA loops were present in functional Ago complexes, we performed functional assays. We constructed sensor plasmids bearing target sequences perfectly complementary to the loop species of several miRNAs. We cotransfected the sensor plasmid with cognate or noncognate miRNA overexpression plasmids in HeLa cells (Fig. 7D). We readily detected repression of loop sensors by pri-let-7a-2, pri-let-7c, and pri-mir-34a, consistent with our immunoprecipitation-Northern analysis and providing direct evidence for the functional regulatory capacity of mammalian loop species.

In summary, a subset of miRNA loops are also loaded to the Argonaute complexes in mammalian cells, suggesting the broad conservation of loop loading. The selectivity of loop loading both in human and fly cells suggests that complex mechanisms operate to select specific miRNA loop species to the effector Argonaute complexes, even though miRNA loop regions generally do not form stable secondary structures.

\section{Discussion}

miRNA loops are a novel source for Argonautedependent RNAs

Studies in the past few years have uncovered an unexpected variety of guide RNA molecules loaded in the Argonaute proteins, and the catalog continues to grow (Yang and Lai 2011). The canonical small RNA substrates for Argonautes that mediate miRNA/siRNA function are 21- to 24-nt ssRNAs that bear 5' monophosphates and derive from small RNA duplexes. Our finding that a subset of miRNA loops are loaded to Argonautes adds a new class of endogenous small regulatory RNA molecules from ssRNA precursors. These findings were unexpected given that synthetic ss-siRNAs were initially reported not to be effective for gene silencing (Elbashir et al. 2001), and in vitro studies indicated that the efficiency of ssRNA loading is significantly lower compared with siRNA duplexes (Martinez et al. 2002; Schwarz et al. 2002). It may be that the modest properties of exogenous ssRNAs may have more to do with their instability as opposed to their inability to be loaded effectively.

More generally, these findings suggest that Argonautes may associate with additional RNAs derived

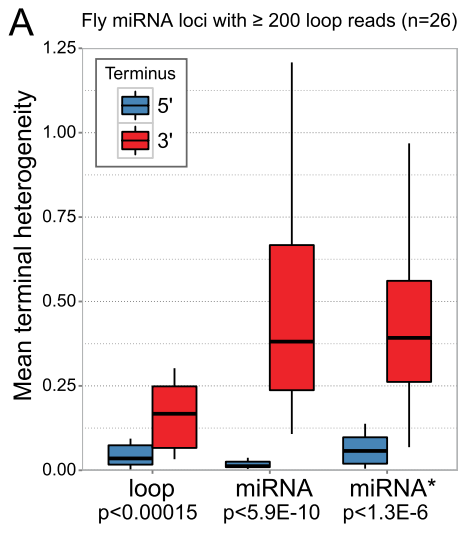

Figure 6. Heterogeneity of mature, star, and loop species. Small RNA reads in fly $(A)$ or mouse $(B)$ libraries mapping to the mature, star, and loop regions were collected, and the heterogeneity at the terminus was calculated as the mean of the absolute distance between the $5^{\prime}$ or $3^{\prime}$ end of an individual read and the most abundant $5^{\prime}$ or $3^{\prime}$ end for each species. Briefly, we allow $6 \mathrm{nt}$ beyond the $5^{\prime}$ and $3^{\prime}$ ends of mature and star species and $6 \mathrm{nt}$ beyond the estimated $5^{\prime}$ and $3^{\prime}$ ends for estimated loop regions. The box plots follow Tukey's standard conventions. The rectangle shows the first to third quartiles, with the thicker horizontal line at the median; whiskers connected to the rectangle extend maximum $1.5 \times$ interquartile range. $P$-values were calculated by the Mann-Whitney $U$-test. 


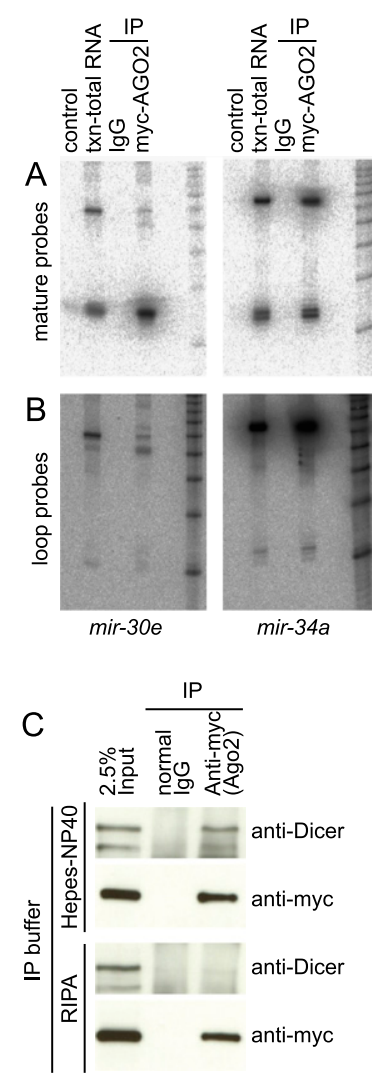

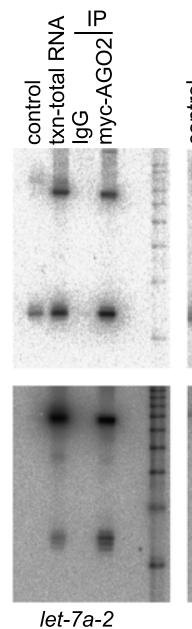
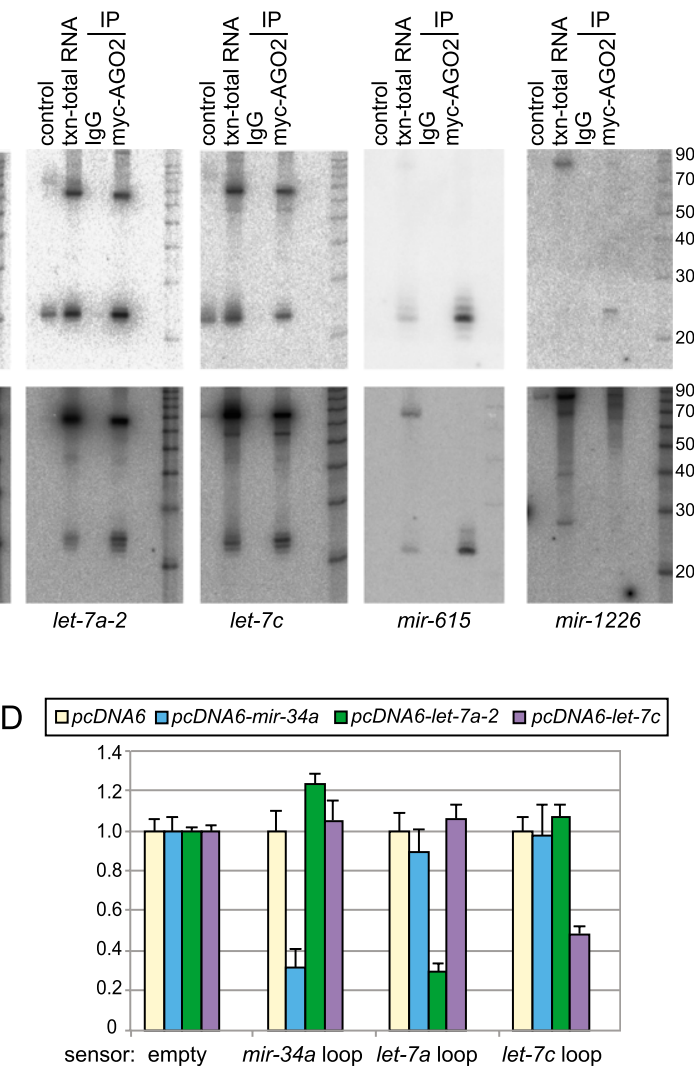

Figure 7. Loading of human miRNA loops to human Ago2. $(A, B)$ The indicated miRNAs were overexpressed under the control of the CMV promoter together with myc-tagged human Ago2 in HeLa cells. The myc-Ago2 complex was purified in RIPA buffer. The indicated miRNA loops (top panels) or mature miRNAs (bottom panels) were detected by Northern blotting. (Lanes 1) Total RNA extracted from HeLa cells transfected with an unrelated miRNA. (Lanes 2) Total RNA extracted from HeLa cells overexpressing the cognate miRNA. (Lanes 3) Control immunoprecipitation using normal rabbit IgG and cell lysate expressing the cognate miRNA. (Lanes 4) Immunoprecipitation using anti-myc antibody and cell lysate expressing the cognate miRNA. Membranes were hybridized with probes against the mature species $(A)$ or the loop species $(B)$. mir-34a, let-7a-2, let-7c, and mir-615 produced detectable miRNA loop species incorporated in the myc-Ago2 complex. $(C)$ The absence of Dicer in the myc-Ago2 complex purified in RIPA buffer. Although Dicer was detectable in the myc-Ago2 complex in a milder condition (Hepes-NP40 buffer; top panels), no coprecipitation was detected in RIPA buffer (bottom panels), which was used for the experiments shown in $A$ and $B$. (D) Luciferase sensor assay to detect miRNA loop activity in human cells. HeLa cells were transfected with mycAgo2 and the designated miRNA overexpression plasmids, together with the luciferase sensors bearing the perfectly complementary target for the miRNA loop species. The luciferase sensors containing the let-7a-2, let-7c, and mir-34a loop targets were repressed when the cognate miRNA genes were overexpressed. from single-stranded precursors. The presence of miRNA loop reads was regarded as a by-product of miRNA biogenesis in previous studies; however, their characteristic cloning patterns with precise $5^{\prime}$ ends and enrichment in Argonaute complexes should have stimulated their functional study. On the other hand, it would be more challenging to identify small regulatory RNA species derived from single-stranded precursors that lack specific ends determined by particular RNases. While such species are usually relegated to the "background" category, our findings should encourage more comprehensive analysis of Argonaute immunoprecipitation libraries to fully understand the range of endogenous guide RNAs.

\section{Endogenous regulatory significance of miRNA loops}

We demonstrated the capacity of miRNA loops to direct target repression in flies and mammals using typical sensor assays that are widely used to assess the function of mature miRNA strands. It was noted earlier that overexpression of the paralogous loci mir-181a-1 and mir-181c caused distinct phenotypes during early T-cell development in mice (Liu et al. 2008). This functional difference was attributed to their distinct loop sequences and led to the notion that pri- or pre-miRNAs may have regulatory roles (Trujillo et al. 2010). However, it is also conceivable that the regulatory activity of loop regions may contribute to the distinct activities of mir-181 genes.

Many miRNA loops are documented to be wellconserved in mammalian species (Michlewski et al. 2008), and Drosophila miRNA gene alignments similarly reveal a number of highly conserved loop regions (Okamura et al. 2008). At least some conserved sequences in miRNA loops represent binding sites for sequence-specific RNAbinding proteins, but this alone cannot explain the high degree of constraint across the length of some miRNA loops. For example, there is only a modest overall sequence similarity between the many let-7 genes whose terminal loops confer selective regulation by the Lin-28 RNAbinding protein (Kim et al. 2010). However, we demonstrated that several well-conserved let-7 gene loops are loaded into Ago complexes (Fig. 7B; Supplemental Fig. S2), potentially suggesting that this feature has been selected across members of this miRNA family. In addition, our demonstration of functional mir-34 loop species in multiple Drosophilids and mammals may imply that this is a conserved feature of mir-34 genes. Our studies provide a rationale to assess whether any loop species contribute to regulatory effects observed in miRNA functional analysis. 


\section{Mechanism of loop loading}

Most mechanistic knowledge regarding how specific RNA species can be preferentially used as guide strands have focused on small RNA duplex properties (Czech and Hannon 2010; Betancur et al. 2012). Our findings reveal that certain single-stranded species are used as endogenous Argonaute guides. Nevertheless, genome-wide analysis shows that loop loading is a highly selective process in both flies and mammals (Fig. 5). Our loop truncation analysis of fly pri-mir-34 indicates that shorter loops are not loaded effectively. Perhaps the propensity of many miRNAs to maintain relatively short loops prevents inappropriate loop loading. It is also known that the MID domain of miRNA-class Argonautes prefers 5' U small RNA species, enforcing the miRNA 5' U bias (Frank et al. 2010; Ameres et al. 2011). Consistent with this, the three most abundant fly miRNA loop species in AGO1 complexes-namely those from mir-34, mir-317, and mir-33-all bear 5' Us. However, the 5' nucleotide and the loop lengths are not the sole determinants of loop loading. Several miRNA loops of appropriate lengths and bearings from highly expressed miRNA hairpins did not accumulate substantially (Fig. 5). Furthermore, we failed to reprogram the terminal loops of mir-34 or mir-1 hairpins to produce the miR-1010 mature sequence and did not detect mir-34 loop activity when it was placed in the loop region of the mir-1 hairpin (Fig. 4C,D). These results suggest the looploading mechanism selects specific species according to the sequences and/or structures of both stems and loops.

Because Argonaute proteins can associate with the dicing machinery (Chendrimada et al. 2005; Maniataki and Mourelatos 2005), it seems plausible that the proximity between the Argonaute and the newly processed loop may augment loading. However, loading of a subset of Drosophila miRNA loops to the AGO2 complex argues against this hypothesis, since the miRNA Dicer (Dcr-1) does not seem to interact with AGO2 (Okamura et al. 2004). Instead, the fact that the two abundant fly miRNA loop species are differentially partitioned between the two Argonautes indicates that ssRNAs are subject to sorting. We further demonstrated that synthetic oligoribonucleotides with miRNA loop sequences could be sorted to specific Argonaute complexes (Fig. 3F). This was unexpected given that sorting of small RNAs to AGO1 and AGO2 was considered to be well explained by duplex structures and 5' nucleotides (Tomari et al. 2007; Ghildiyal et al. 2008, 2010; Czech et al. 2009; Okamura et al. 2009). AGO1 selects small RNA duplexes with central mismatches and more strictly prefers 5' U small RNA species, whereas AGO2 selects duplexes with perfectly basepairing central regions and efficiently load $5^{\prime} \mathrm{U}$ and $5^{\prime}$ C small RNA species. Our genome-wide analysis in flies indicates that AGO1 exhibits stricter selectivity for specific loop species than AGO2 (Fig. 5A,B). Sorting of miRNA loop species does not seem to correlate with the sorting of mature miRNAs (Fig. 1B), suggesting that miRNA loop sorting occurs independently of miRNA duplex sorting. Unlike duplex sorting, the siRNA-loading complex does not seem to have a role in loop loading (Fig. 5B). The recapitulation of loop sorting by our in vitro assay suggests that the sorting signal lies in the loop sequences or structures (Fig. 3D-F). Supporting this notion, we could recapitulate sorting of the loop sequences using preprocessed synthetic miRNA loops (Fig. 3F). We suspect that the preferences for the specific loop species reflect the nature of Argonaute proteins.

Our results open an experimental system to study the sorting and loading of endogenous ssRNAs. Of note is the observation that inhibition or mutation of exonucleases causes accumulation of miRNA loops in Arabidopsis (Gy et al. 2007), although it remains to be seen whether these loops are incorporated in the functional complex. Loop loading may become more prevalent under certain conditions in which cells exhibit lower ribonuclease activity. Further analysis of loop loading may be relevant toward improving the activity of synthetic ss-siRNAs, which have only recently been shown to be effective in vivo using appropriate nucleotide modifications (Chorn et al. 2012; Lima et al. 2012; Yu et al. 2012).

\section{Materials and methods}

\section{Library analysis}

Small RNA libraries were constructed as described previously (Czech et al. 2008) using the AGO1 or Flag-AGO2 complex immunopurified from $\mathrm{S} 2-\mathrm{R}^{+}$cells expressing Flag-AGO2 protein by anti-AGO1 or anti-Flag antibody. In addition to the two new libraries, we analyzed 250 fly and 320 mouse small RNA data sets from NCBI Gene Expression Omnibus (GEO)/Sequence Read Archive (SRA), as summarized in Supplemental Table S3; relevant ancillary information was captured from GEO and SRA to help organize the data in local databases. Our preference was to use raw data in the form of fastq files when available. Those reads in which the adaptor was successfully clipped were then filtered by sizes $>14 \mathrm{nt}$ and $<35 \mathrm{nt}$ and collapsed by total read count to minimize the amount of redundant information. Bowtie was then used for genome mapping to D. melanogaster or Mus musculus (dm3 or $\mathrm{mm} 9$ builds, respectively). Bowtie's parameters were to allow only perfect matches to the genome $-\mathrm{v} 0$. Unmapped reads were saved to a separate file for later processing at the end of the pipeline.

To identify miRNA loop regions, we first extracted the most abundant reads from the $5^{\prime}$ and $3^{\prime}$ arms of the miRNA and defined the loop ends based on the ends of the most abundant mature and star reads, taking 3' 2-nt overhangs in consideration. We allowed reads to overlap to the next windows as long as $6 \mathrm{nt}$ and classified the reads as mature, star, and loop reads. Only loop candidate reads that occur within the genome two or fewer times were considered. To analyze small RNA fragments mapping to rRNAs and RpL mRNAs, we extracted reads mapping to $18 \mathrm{~S}$ and $28 \mathrm{~S}$ rRNAs and the 45 ribosomal large subunit protein mRNAs annotated in FlyBase (listed in Supplemental Table S1, "RpL genes" tab). For the scatter plots shown in Figure 3, we counted reads from each loop or hairpin (mature + star + loop) region, and read counts were normalized by the number of total miRNA reads. The libraries used for each figure are listed in Supplemental Table S3.

\section{Luciferase assay}

Luciferase assays were carried out as previously described (Okamura et al. 2007; Yang et al. 2011). The luciferase sensor plasmids were constructed by inserting two-repeat target se- 
quences of miRNAs or miRNA loops in the NotI and XhoI sites of a modified psiCHECK plasmid (Okamura et al. 2007). miRNA overexpression plasmids were constructed by inserting genomic fragments amplified by PCR in pcDNA6 (for human miRNAs) or pDsRed (for fly miRNAs). For the luciferase assays in HeLa cells, pcDNA-myc-hAgo2 plasmid was cotransfected. All values were normalized against the values with the psiCHECK empty sensor values and then normalized again with the pDsRed empty vector values. Oligonucleotides used for the sensor constructs are listed in Supplemental Table S4.

\section{Immunoprecipitation and Northern blotting}

Immunoprecipitation was performed using a previously described protocol (Okamura et al. 2011). S2- $\mathrm{R}^{+}$cells expressing Flag-AGO2 or dissected heads were lysed in RIPA buffer ( $1 \times$ PBS, $0.5 \%$ NP- 40 , $0.5 \%$ deoxycholate, $0.1 \%$ SDS, $1 \times$ Complete EDTA free [Roche]) or Hepes-NP40 buffer (30 mM HEPES at $\mathrm{pH} 7.4,100 \mathrm{mM}$ sodium chloride, $2 \mathrm{mM}$ magnesium acetate, $5 \mathrm{mM}$ DTT, $0.1 \%$ NP40), respectively. HeLa cells were transiently transfected with miRNA overexpression plasmids along with the pcDNA-myc-hAgo2 plasmid as described previously (Yang et al. 2010). The mycAgo2 complex was purified with anti-myc (Santa Cruz Biotechnology, A-14) in RIPA or Hepes-NP40 buffer. RNA was extracted from immunoprecipitation and input samples for Northern blotting analysis. Northern blotting was performed as previously described (Okamura et al. 2007). The probe sequences are listed in Supplemental Table S4.

\section{RNAi}

S2- $\mathrm{R}^{+}$cells were stably transfected with a plasmid containing a metallothionein promoter fused to mir-34 gene and soaked with dsRNAs for $8 \mathrm{~d}$ as previously described (Okamura et al. 2009). mir-34 expression was induced by adding $2 \mathrm{mM} \mathrm{CuSO}_{4}$ on day 7 .

\section{In vitro loading assay}

We modified the in vitro small RNA processing assay protocol to monitor loading of miRNA loops. Radiolabeled pre-miRNAs were prepared by ligating two oligoribonucleotides according to the previously published protocol (Kurschat et al. 2005). The oligoribonucleotides used for pre-miRNA preparation are shown in Supplemental Table S4. In brief, the RNA oligonucleotide corresponding either the $5^{\prime}$ or $3^{\prime}$ half was labeled at the $5^{\prime}$ end with ${ }^{32} \mathrm{P}$ and mixed with the partner RNA oligonucleotide phosphorylated at the $5^{\prime}$ end and the bridge DNA oligonucleotide. These oligonucleotides were annealed to form a DNA-RNA hybrid, and the RNA molecules were ligated by T4 DNA ligase. After digesting the DNA strand, the ligated product was gelpurified and used for the in vitro loading assay. Approximately $1 \times 10^{4}$ to $5 \times 10^{4} \mathrm{cpm}$ (counts per minute) of the labeled premiRNAs were incubated in $320 \mu \mathrm{L}$ of S2- $\mathrm{R}^{+}$cell lysate, and after the incubation, the AGO1 complex was purified by immunoprecipitation using anti-AGO1 antibody (AbCam) immobilized on DynaBeads (Invitrogen) in RIPA buffer. Coprecipitated RNA was purified by phenol/chloroform/isoamylalcohol extraction and separated on $15 \%$ denaturing gels. Separated RNA was transferred onto nylon membrane (Genescreen plus) and analyzed by PhosphorImager. For the Hsp70 inhibition experiments, we added $1 \mathrm{mM}$ PES to the lysate prior to the addition of the labeled precursor.

\section{Acknowledgments}

We thank Gregory Hannon, Joanne Yew, and Jidong Liu for reagents, and Rui Yi for small RNA library data. We thank many researchers that deposited their small RNA data in public databases. Work in E.C.L.'s group was supported by the Burroughs Wellcome Fund and the National Institutes of Health/National Institute of General Medical Sciences (R01-GM083300). K.O. was a research fellow of the Japan Society for the Promotion of Science. K.O. and his group were supported by the National Research Foundation (NRF2011NRF-NRFF001-042). The content is solely the responsibility of the authors and does not necessarily represent the official views of these agencies.

\section{Note added in proof}

The small RNA sequencing data were deposited at Short Read Archive under accession SRP018995. While this article was in production, another study (Winter et al. 2013) came to our attention describing functional miRNA loop species in human cells.

\section{References}

Ameres SL, Horwich MD, Hung JH, Xu J, Ghildiyal M, Weng Z, Zamore PD. 2010. Target RNA-directed trimming and tailing of small silencing RNAs. Science 328: 1534-1539.

Ameres SL, Hung JH, Xu J, Weng Z, Zamore PD. 2011. Target RNA-directed tailing and trimming purifies the sorting of endo-siRNAs between the two Drosophila Argonaute proteins. RNA 17: 54-63.

Azuma-Mukai A, Oguri H, Mituyama T, Qian ZR, Asai K, Siomi H, Siomi MC. 2008. Characterization of endogenous human Argonautes and their miRNA partners in RNA silencing. Proc Natl Acad Sci 105: 7964-7969.

Bajan S, Hutvagner G. 2011. Another 'loophole' in miRNA processing. Mol Cell 44: 345-347.

Berezikov E, Robine N, Samsonova A, Westholm JO, Naqvi A, Hung JH, Okamura K, Dai Q, Bortolamiol-Becet D, Martin R, et al. 2011. Deep annotation of Drosophila melanogaster microRNAs yields insights into their processing, modification, and emergence. Genome Res 21: 203-215.

Betancur JG, Yoda M, and Tomari Y. 2012. miRNA-like duplexes as RNAi triggers with improved specificity. Front Genet 3: 127.

Burroughs AM, Ando Y, de Hoon MJ, Tomaru Y, Nishibu T, Ukekawa R, Funakoshi T, Kurokawa T, Suzuki H, Hayashizaki Y, et al. 2010. A comprehensive survey of $3^{\prime}$ animal miRNA modification events and a possible role for $3^{\prime}$ adenylation in modulating miRNA targeting effectiveness. Genome Res 20: 1398-1410.

Chendrimada TP, Gregory RI, Kumaraswamy E, Norman J, Cooch N, Nishikura K, Shiekhattar R. 2005. TRBP recruits the Dicer complex to Ago2 for microRNA processing and gene silencing. Nature 436: 740-744.

Chorn G, Klein-McDowell M, Zhao L, Saunders MA, Flanagan WM, Willingham AT, Lim LP. 2012. Single-stranded microRNA mimics. RNA 18: 1796-1804.

Czech B, Hannon GJ. 2010. Small RNA sorting: Matchmaking for Argonautes. Nat Rev Genet 12: 19-31.

Czech B, Malone CD, Zhou R, Stark A, Schlingeheyde C, Dus M, Perrimon N, Kellis M, Wohlschlegel J, Sachidanandam R, et al. 2008. An endogenous siRNA pathway in Drosophila. Nature 453: 798-802.

Czech B, Zhou R, Erlich Y, Brennecke I, Binari R, Villalta C, Gordon A, Perrimon N, Hannon GJ. 2009. Hierarchical rules for Argonaute loading in Drosophila. Mol Cell 36: 445-456.

de Wit E, Linsen SE, Cuppen E, Berezikov E. 2009. Repertoire and evolution of miRNA genes in four divergent nematode species. Genome Res 19: 2064-2074. 
Elbashir SM, Lendeckel W, Tuschl T. 2001. RNA interference is mediated by 21- and 22-nucleotide RNAs. Genes Dev 15: 188-200.

Elkayam E, Kuhn CD, Tocilj A, Haase AD, Greene EM, Hannon GJ, Joshua-Tor L. 2012. The structure of human Argonaute-2 in complex with miR-20a. Cell 150: 100-110.

Ender C, Krek A, Friedlander MR, Beitzinger M, Weinmann L, Chen W, Pfeffer S, Rajewsky N, Meister G. 2008. A human snoRNA with microRNA-like functions. Mol Cell 32: 519-528.

Flynt AS, Lai EC. 2008. Biological principles of microRNAmediated regulation: Shared themes amid diversity. Nat Rev Genet 9: 831-842.

Forstemann K, Horwich MD, Wee L, Tomari Y, Zamore PD. 2007. Drosophila microRNAs are sorted into functionally distinct argonaute complexes after production by dicer-1. Cell 130: $287-297$.

Frank F, Sonenberg N, Nagar B. 2010. Structural basis for 5 '-nucleotide base-specific recognition of guide RNA by human AGO2. Nature 465: 818-822.

Ghildiyal M, Seitz H, Horwich MD, Li C, Du T, Lee S, Xu J, Kittler EL, Zapp ML, Weng Z, et al. 2008. Endogenous siRNAs derived from transposons and mRNAs in Drosophila somatic cells. Science 320: 1077-1081.

Ghildiyal M, Xu J, Seitz H, Weng Z, Zamore PD. 2010. Sorting of Drosophila small silencing RNAs partitions microRNA* strands into the RNA interference pathway. RNA 16: 43-56.

Gy I, Gasciolli V, Lauressergues D, Morel JB, Gombert J, Proux F, Proux C, Vaucheret H, Mallory AC. 2007. Arabidopsis FIERY1, XRN2, and XRN3 are endogenous RNA silencing suppressors. Plant Cell 19: 3451-3461.

Han BW, Hung JH, Weng Z, Zamore PD, Ameres SL. 2011. The $3^{\prime}$-to-5' exoribonuclease Nibbler shapes the $3^{\prime}$ ends of microRNAs bound to Drosophila Argonaute1. Curr Biol 21: 1878-1887.

He M, Liu Y, Wang X, Zhang MQ, Hannon GJ, Huang ZJ. 2012. Cell-type-based analysis of microRNA profiles in the mouse brain. Neuron 73: 35-48.

Horwich MD, Li C, Matranga C, Vagin V, Farley G, Wang P, Zamore PD. 2007. The Drosophila RNA methyltransferase, DmHen1, modifies germline piRNAs and single-stranded siRNAs in RISC. Curr Biol 17: 1265-1272.

Iki T, Yoshikawa M, Nishikiori M, Jaudal MC, MatsumotoYokoyama E, Mitsuhara I, Meshi T, Ishikawa M. 2010. In vitro assembly of plant RNA-induced silencing complexes facilitated by molecular chaperone HSP90. Mol Cell 39: 282-291.

Ishizu H, Siomi H, Siomi MC. 2012. Biology of PIWI-interacting RNAs: New insights into biogenesis and function inside and outside of germlines. Genes Dev 26: 2361-2373.

Iwasaki S, Kobayashi M, Yoda M, Sakaguchi Y, Katsuma S, Suzuki T, Tomari Y. 2010. Hsc70/Hsp90 chaperone machinery mediates ATP-dependent RISC loading of small RNA duplexes. Mol Cell 39: 292-299.

Johnston M, Geoffroy MC, Sobala A, Hay R, Hutvagner G. 2010. HSP90 protein stabilizes unloaded argonaute complexes and microscopic P-bodies in human cells. Mol Biol Cell 21: 1462 1469.

Kawamata T, Tomari Y. 2010. Making RISC. Trends Biochem Sci 35: 368-376.

Kawamata T, Seitz H, Tomari Y. 2009. Structural determinants of miRNAs for RISC loading and slicer-independent unwinding. Nat Struct Mol Biol 16: 953-960.

Kawamura Y, Saito K, Kin T, Ono Y, Asai K, Sunohara T, Okada T, Siomi MC, Siomi H. 2008. Drosophila endogenous small RNAs bind to Argonaute2 in somatic cells. Nature 453: 793-797.

Khvorova A, Reynolds A, Jayasena SD. 2003. Functional siRNAs and miRNAs exhibit strand bias. Cell 115: 209-216.
Kim VN, Han J, Siomi MC. 2009. Biogenesis of small RNAs in animals. Nat Rev Mol Cell Biol 10: 126-139.

Kim YK, Heo I, Kim VN. 2010. Modifications of small RNAs and their associated proteins. Cell 143: 703-709.

Kurschat WC, Muller J, Wombacher R, Helm M. 2005. Optimizing splinted ligation of highly structured small RNAs. RNA 11: 1909-1914.

Lima WF, Prakash TP, Murray HM, Kinberger GA, Li W, Chappell AE, Li CS, Murray SF, Gaus H, Seth PP, et al. 2012. Singlestranded siRNAs activate RNAi in animals. Cell 150: 883-894.

Liu Q, Rand TA, Kalidas S, Du F, Kim HE, Smith DP, Wang X. 2003. R2D2, a bridge between the initiation and effector steps of the Drosophila RNAi pathway. Science 301: 1921-1925.

Liu J, Carmell MA, Rivas FV, Marsden CG, Thomson JM, Song JJ, Hammond SM, Joshua-Tor L, Hannon GJ. 2004. Argonaute 2 is the catalytic engine of mammalian RNAi. Science 305: 1437-1441.

Liu J, Valencia-Sanchez MA, Hannon GJ, Parker R. 2005. MicroRNA-dependent localization of targeted mRNAs to mammalian P-bodies. Nat Cell Biol 7: 719-723.

Liu G, Min H, Yue S, Chen CZ. 2008. Pre-miRNA loop nucleotides control the distinct activities of mir-181a-1 and mir-181c in early T cell development. PLOS ONE 3: e3592.

Liu N, Abe M, Sabin LR, Hendriks GJ, Naqvi AS, Yu Z, Cherry S, Bonini NM. 2011. The exoribonuclease Nibbler controls 3' end processing of microRNAs in Drosophila. Curr Biol 21: 1888-1893.

Maniataki E, Mourelatos Z. 2005. A human, ATP-independent, RISC assembly machine fueled by pre-miRNA. Genes Dev 19: 2979-2990.

Marques JT, Kim K, Wu PH, Alleyne TM, Jafari N, Carthew RW. 2010. Loqs and R2D2 act sequentially in the siRNA pathway in Drosophila. Nat Struct Mol Biol 17: 24-30.

Martinez J, Patkaniowska A, Urlaub H, Luhrmann R, Tuschl T. 2002. Single-stranded antisense siRNAs guide target RNA cleavage in RNAi. Cell 110: 563-574.

Maurin T, Cazalla D, Yang JS, Bortolamiol-Becet D, Lai EC. 2012. RNase III-independent microRNA biogenesis in mammalian cells. RNA 18: $2166-2173$.

Meister G, Landthaler M, Patkaniowska A, Dorsett Y, Teng G, Tuschl T. 2004. Human Argonaute2 mediates RNA cleavage targeted by miRNAs and siRNAs. Mol Cell 15: 185-197.

Michlewski G, Guil S, Semple CA, Caceres JF. 2008. Posttranscriptional regulation of miRNAs harboring conserved terminal loops. Mol Cell 32: 383-393.

Miyoshi K, Tsukumo H, Nagami T, Siomi H, Siomi MC. 2005. Slicer function of Drosophila Argonautes and its involvement in RISC formation. Genes Dev 19: 2837-2848.

Miyoshi T, Takeuchi A, Siomi H, Siomi MC. 2011. A direct role for Hsp90 in pre-RISC formation in Drosophila. Nat Struct Mol Biol 18: 516.

Nakanishi K, Weinberg DE, Bartel DP, Patel DJ. 2012. Structure of yeast Argonaute with guide RNA. Nature 486: 368-374.

Okamura K. 2012. Diversity of animal small RNA pathways and their biological utility. Wiley Interdiscip Rev RNA 3: 351368.

Okamura K, Ishizuka A, Siomi H, Siomi MC. 2004. Distinct roles for Argonaute proteins in small RNA-directed RNA cleavage pathways. Genes Dev 18: 1655-1666.

Okamura K, Hagen JW, Duan H, Tyler DM, Lai EC. 2007. The mirtron pathway generates microRNA-class regulatory RNAs in Drosophila. Cell 130: 89-100.

Okamura K, Phillips MD, Tyler DM, Duan H, Chou YT, Lai EC. 2008. The regulatory activity of microRNA* species has substantial influence on microRNA and 3' UTR evolution. Nat Struct Mol Biol 15: 354-363. 
Okamura K, Liu N, Lai EC. 2009. Distinct mechanisms for microRNA strand selection by Drosophila Argonautes. Mol Cell 36: 431-444.

Okamura K, Robine N, Liu Y, Liu Q, Lai EC. 2011. R2D2 organizes small regulatory RNA pathways in Drosophila. Mol Cell Biol 31: 884-896.

Pelaez N, Carthew RW. 2012. Biological robustness and the role of microRNAs: A network perspective. Curr Top Dev Biol 99: 237-255.

Rivas FV, Tolia NH, Song JJ, Aragon JP, Liu J, Hannon GJ, JoshuaTor L. 2005. Purified Argonaute 2 and an siRNA form recombinant human RISC. Nat Struct Mol Biol 12: 340-349.

Ruby JG, Stark A, Johnston WK, Kellis M, Bartel DP, Lai EC. 2007. Evolution, biogenesis, expression, and target predictions of a substantially expanded set of Drosophila microRNAs. Genome Res 17: 1850-1864.

Ruwe H, Schmitz-Linneweber C. 2012. Short non-coding RNA fragments accumulating in chloroplasts: Footprints of RNA binding proteins? Nucleic Acids Res 40: 3106-3116.

Schwarz DS, Hutvagner G, Haley B, Zamore PD. 2002. Evidence that siRNAs function as guides, not primers, in the Drosophila and human RNAi pathways. Mol Cell 10: 537-548.

Schwarz DS, Hutvagner G, Du T, Xu Z, Aronin N, Zamore PD. 2003. Asymmetry in the assembly of the RNAi enzyme complex. Cell 115: 199-208.

Seitz H, Ghildiyal M, Zamore PD. 2008. Argonaute loading improves the $5^{\prime}$ precision of both MicroRNAs and their miRNA strands in flies. Curr Biol 18: 147-151.

Tomari Y, Matranga C, Haley B, Martinez N, Zamore PD. 2004. A protein sensor for siRNA asymmetry. Science 306: 13771380.

Tomari Y, Du T, Zamore PD. 2007. Sorting of Drosophila small silencing RNAs. Cell 130: 299-308.

Trujillo RD, Yue SB, Tang Y, O'Gorman WE, Chen CZ. 2010. The potential functions of primary microRNAs in target recognition and repression. EMBO I 29: 3272-3285.

Wang D, Zhang Z, O'Loughlin E, Lee T, Houel S, O'Carroll D, Tarakhovsky A, Ahn NG, Yi R. 2012. Quantitative functions of Argonaute proteins in mammalian development. Genes Dev 26: 693-704.

Westholm JO, Ladewig E, Okamura K, Robine N, Lai EC. 2012. Common and distinct patterns of terminal modifications to mirtrons and canonical microRNAs. RNA 18: 177-192.

Winter J, Link S, Witzigmann D, Hildenbrand C, Previti C, Diederichs S. 2013. Loop-miRs: Active microRNAs generated from single-stranded loop regions. Nucl Acids Res (in press).

Wu H, Neilson JR, Kumar P, Manocha M, Shankar P, Sharp PA, Manjunath N. 2007. miRNA profiling of naïve, effector and memory CD8 T cells. PLOS ONE 2: e1020.

Yang IS, Lai EC. 2011. Alternative miRNA biogenesis pathways and the interpretation of core miRNA pathway mutants. Mol Cell 43: 892-903.

Yang JS, Maurin T, Robine N, Rasmussen KD, Jeffrey KL, Chandwani R, Papapetrou EP, Sadelain M, O'Carroll D, Lai EC. 2010. Conserved vertebrate mir-451 provides a platform for Dicer-independent, Ago2-mediated microRNA biogenesis. Proc Natl Acad Sci 107: 15163-15168.

Yang JS, Phillips MD, Betel D, Mu P, Ventura A, Siepel AC, Chen KC, Lai EC. 2011. Widespread regulatory activity of vertebrate microRNA* species. RNA 17: 312-326.

Yu D, Pendergraff H, Liu J, Kordasiewicz HB, Cleveland DW, Swayze EE, Lima WF, Crooke ST, Prakash TP, Corey DR. 2012. Single-stranded RNAs use RNAi to potently and alleleselectively inhibit mutant huntingtin expression. Cell 150: 895-908. 


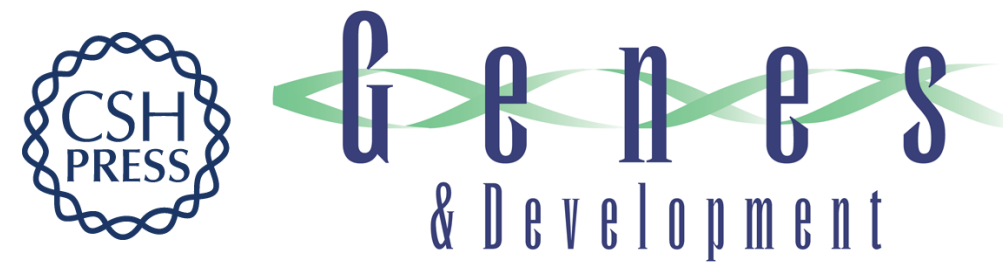

\section{Functional small RNAs are generated from select miRNA hairpin loops in flies and mammals}

Katsutomo Okamura, Erik Ladewig, Li Zhou, et al.

Genes Dev. 2013, 27: originally published online March 27, 2013

Access the most recent version at doi:10.1101/gad.211698.112

\section{Supplemental http://genesdev.cshlp.org/content/suppl/2013/03/20/gad.211698.112.DC1 Material}

References This article cites 74 articles, 27 of which can be accessed free at: http://genesdev.cshlp.org/content/27/7/778.full.html\#ref-list-1

\section{License}

Email Alerting

Receive free email alerts when new articles cite this article - sign up in the box at the top Service

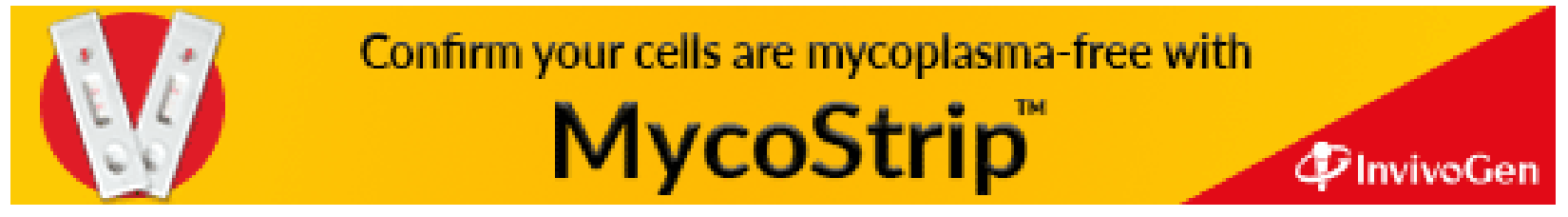

